\title{
Alcances y retos de la Neumología Intervencionista
}

\section{Scope and challenges of Interventional Pulmonology}

ISABEL PaLAcios ORTEga, MD.

En los últimos años, la Neumología ha pasado de la evaluación clínica juiciosa propia del método de Osler, a la práctica activa de procedimientos mínimamente invasivos que imponen el reto de transitar en ese delgado límite entre la clínica y la cirugía, sin el uso del bisturí, apenas un sutil acercamiento a Barré, con la firme convicción de la necesidad de un trabajo articulado con el grupo de Cirugía de tórax.

Desde Gustav Killian, padre de la broncoscopia, y luego con Chevalier Jackson, ambos laringólogos, fue posible la visualización de la vía aérea, no obstante mediante broncoscopia rígida, procedimiento inicialmente realizado bajo anestesia local, y con todas las incomodidades que implicaba $(1,2)$.

Luego, gracias al broncoscopio flexible de Ikeda, no sólo se hizo más amable el procedimiento para el paciente (3), sino más práctico, accesible y fácil de manipular para el neumólogo; empero con el abandono de la broncoscopia rígida, llegó el de la instrucción de la misma, hecho que conudjo a que en la actualidad sólo el 4\% de los neumólogos la empleen con experticia (4). Este es un procedimiento útil para el manejo de la vía aérea difícil, las estenosis traqueales, el retiro de cuerpos extraños, entre otros, de ahí que sea necesario el rescate de la técnica y el entrenamiento de neumólogos intervencionistas.

Mientras por un lado se rescata la instrucción en broncoscopia rígida, por otro, con la aparición de las endoprótesis expansibles para el manejo de estenosis relacionadas con neoplasias, se facilita su aplicación mediante broncoscopia flexible. Inicialmente metálicas y ahora con nuevos materiales y también recubiertos, las endoprótesis expansibles se han convertido en una opción de tratamiento sencilla (5), aunque bajo el seguimiento de indicaciones estrictas según sus efectos adversos y la polémica sobre su uso en pacientes con cáncer dadas las dificultades en su retiro.

Desde el punto de vista diagnóstico, antes solo útil en la búsqueda etiológica de infecciones broncopulmonares, ahora el espectro de la Neumología intervencionista va más allá de las paredes traqueobronquiales, logrando alcanzar ganglios mediastinales o masas que antes eran inaccesibles, de no hacerse mediante procedimientos quirúrgicos como la mediastinoscopia (6). Inicialmente utilizada para la estadificación del
Neumóloga Intervencionista (Interventional Pulmonary Fellowship for Latinoamerican). Centro Médico Imbanaco. Cali, Colombia.

Correspondencia: Isabel Palacios Ortega. Correo electrónico: isabelpalaciosmd@ gmail.com

Recibido: 12-12-2013. Aceptado: 31-12-2013. 
cáncer, ahora los avances en anatomía patológica han permitido que incluso el espécimen recolectado por punción por endosonografía bronquial (EBUS), también pueda utilizarse para pruebas moleculares que hacen la diferencia en el tratamiento del cáncer.

Por otra parte, lesiones parenquimatosas y nódulos pulmonares periféricos antes inaccesibles, ahora son posibles gracias a la toma de muestras bajo visión en tiempo real por endosonografía radial (7), al igual que con la aparición de sistemas de navegación que simulan tecnologías como GPS, utilizadas para la orientación en la vida real.

El espectro se amplía aún más cuando la Neumología Intervencionista permite aportar al manejo de patologías como el asma y la EPOC, cuando los tratamientos farmacológicos han agotado su capacidad, con el adecuado conocimiento de nuevas tecnologías como la termoplastia bronquial y las válvulas de reducción de volumen, pero sobre todo con la identificación del paciente indicado con miras a mejorar sustancialmente su calidad de vida $(8,9)$.

Con la reaparición de la Neumología Intervencionista surgen nuevos retos que implican no sólo el entrenamiento en estas nuevas tecnologías, sino el completo conocimiento de la anatomía, la identificación del paciente correcto, el conocimiento de sus complicaciones así como el manejo de las mismas, y la conciencia de la necesidad permanente de trabajo engranado en equipo con el grupo de Cirugía de tórax, Oncología y Otorrinolaringología. Posiblemente el principal reto será no desligarnos de la clínica y del manejo directo del paciente.

\section{Bibliografía}

1. Prowse SJ, Makura Z. Gustav Killian: beyond his dehiscence. J Laryngol Otol. 2012;126:1164-8.

2. Boyd AD. Chevalier Jackson: the father of American bronchoesophagoscopy. Ann Thorac Surg. 1994;57:502-5.

3. Ikeda S, Yanai N, Ishikawa S. Flexible bronchofiberscope. Keio J Med. 1968;17:1-16.

4. Diaz-Mendoza, JI. Kvale, PA. The rigid bronchoscope: a pulmonologist's forgotten tool? CHEST Physician. 2011. Disponible en: http://69.36.35.38/accp/article/chest-physician/rigid-bronchoscope-pulmonologist $\%$ E2\%80\%99s-\%E2\%80\%98forgottentool\%E2\%80\%99. [Consultado 12 Dic 2013].

5. Lee P, Kupeli E, Mehta AC. Airway stents. Clin Chest Med. 2010;31:141-50.

6. Detterbeck FC, Postmus PE, Tanoue LT. The stage classification of lung cancer: Diagnosis and management of lung cancer, 3rd. ed: American College of Chest Physicians evidence-based clinical practice guidelines. Chest. 2013;143(5 Suppl):191S-210S.

7. Herth FJ, Ernst A, Becker HD. Endobronchial ultrasound-guided transbronchial lung biopsy in solitary pulmonary nodules and peripheral lesions. Eur Respir J. 2002;20:972-4.

8. Sheshadri A, Castro M, Chen A. Bronchial thermoplasty: a novel therapy for severe asthma. Clin Chest Med. 2013;34:437-44.

9. Gasparini S, Zuccatosta L, Bonifazi M, Bolliger CT. Bronchoscopic treatment of emphysema: state of the art. Respiration. 2012;84:250-63. 